Research Paper

\title{
Metabolic Determinants and Anthropometric Indicators Impact Clinical-pathological Features in Epithelial Ovarian Cancer Patients
}

\author{
Patrizia Vici ${ }^{*}$, Laura Pizzuti ${ }^{*}$, Luigi Di Lauro ${ }^{1}$, Laura Conti2 ${ }^{2}$, Chiara Mandoj ${ }^{2}$, Anna Antenucci2 ${ }^{2}$ Giovanna \\ Digiesi ${ }^{2}$, Domenico Sergi ${ }^{1}$, Antonella Amodio, Paolo Marchetti ${ }^{3}$, Francesca Sperati ${ }^{4}$, Mario Valle ${ }^{5}$, Alfredo \\ Garofalo5, Enrico Vizza6, Giacomo Corrado ${ }^{6}$, Cristina Vincenzoni 6 , Federica Tomao ${ }^{7}$, Ramy Kayal ${ }^{8}$, \\ Annalise Marsella 8 , Mariantonia Carosi ${ }^{9}$, Barbara Antoniani ${ }^{9}$, Antonio Giordano ${ }^{10,11}$, Marcello \\ Maugeri-Saccà ${ }^{*}, 12$ and Maddalena Barba ${ }^{* 1,12} \bowtie$
}

1. Division of Medical Oncology 2, Regina Elena National Cancer Institute, Rome, Italy

2. Division of Clinical Pathology, Regina Elena National Cancer Institute, Rome, Italy

3. Oncology Unit, Sant' Andrea Hospital, La Sapienza University of Rome, Italy

4. Biostatistics Unit-Scientific Direction, Regina Elena National Cancer Institute, Rome, Italy

5. General Surgery, Regina Elena National Institute, Rome, Italy

6. Gynecological Oncology, Regina Elena National Cancer Institute, Rome, Italy

7. Department of Gynecologic Oncology, University "Sapienza", Viale del Policlinico 155, 00161 Rome, Italy

8. Department of Radiology, Regina Elena National Cancer Institute, Rome, Italy

9. Department of Pathology, Regina Elena National Cancer Institute, Rome, Italy

10. Sbarro Institute for Cancer Research and Molecular Medicine e del Center for Biotechnology, College of Science and Technology, Temple University, Philadelphia, PA, USA

11. Department of Medicine, Surgery and Neurosciences, University of Siena, Siena, Italy

12. Scientific Direction, Regina Elena National Cancer Institute, Rome, Italy.

*Equal contributors

$\triangle$ Corresponding author: Maddalena Barba, MD, PhD. Division of Medical Oncology 2-Scientific Direction, Regina Elena National Cancer Institute, Via Elio Chianesi 53, 0144 Rome, Italy. Email address: maddalena.barba@gmail.com; Landline: +390652665419; Fax: +390652666249

(C) Ivyspring International Publisher. Reproduction is permitted for personal, noncommercial use, provided that the article is in whole, unmodified, and properly cited. See http://ivyspring.com/terms for terms and conditions.

Received: 2015.08.17; Accepted: 2015.12.08; Published: 2016.02.10

\begin{abstract}
Background: Over the last twenty years, the efforts of the scientific community devoted to the comprehension and treatment of ovarian cancer have remained poorly remunerative, with the case-fatality ratio of this disease remaining disappointedly high. Limited knowledge of the basic principles regulating ovarian carcinogenesis and factors impacting the course of disease may significantly impair our ability to intervene in early stages and lessen our expectations in terms of treatment outcomes. In the present study, we sought to assess whether metabolic factors and anthropometric indicators, i.e., pre-treatment fasting glucose and body mass index, are associated with renown cancer related prognostic factors such as tumour stage and grade at diagnosis.
\end{abstract}

Materials and Methods: Study participants were 147 women diagnosed with epithelial ovarian cancer and treated with platinum based regimens and/or surgery at the Regina Elena National Cancer Institute of Rome, Italy. Glucose levels were assessed at the institutional laboratories on venous blood collected in overnight fasting conditions and prior to any therapeutic procedure. Stage was coded according to the FIGO staging system based on the results of the diagnostic workup, while tumour grade was locally assessed by an expert pathologist. Participants' characteristics were descriptively analyzed for the overall study population and in a subgroup of 70 patients for whom data on body mass index (BMI) were available. FIGO stage and grade were compared by categories of pre-treatment fasting glucose defined upon the median value, i.e., $89 \mathrm{mg} / \mathrm{dl}$. The association of interest was tested in regression models including BMI.

Results: For the overall study population, patients in the lowest category of fasting glucose were significantly more likely to exhibit a FIGO stage III-IV at diagnosis compared with their counterpart in the highest glucose category (81.3 vs $66.7 \%$, p: 0.021 ). Subgroup analysis in 70 patients with BMI data 
confirmed this association ( 81.5 vs 55.8 , p: 0.049$)$, which remained significant when tested in regression models including BMI (OR: $0.2895 \% \mathrm{Cl}$ 0.086-0.89, p: 0.031). No relevant evidence emerged when testing the association between fasting glucose and tumour grade.

Conclusions: In patients diagnosed with epithelial ovarian cancer, pre-treatment glucose levels appear to be inversely associated with FIGO stage. Further studies are warranted to eventually confirm and correctly interpret the implications of this novel finding.

Key words: epithelial ovarian cancer, fasting glucose, body mass index, FIGO stage, tumour grade

\section{Background}

Despite intensive efforts from the scientific community, statistics for ovarian cancer have remained fairly stable in European countries over the past two decades, with the contribution paid to this disease in terms of specific mortality being still unacceptably high. Major difficulties in diagnosis and therapeutic management might at least partly arise from limited knowledge of the basic principles regulating ovarian carcinogenesis and factors impacting the course of disease. When translated to the clinical setting, such limitations may significantly impair our ability to intervene in early disease stages and lessen our expectations in terms of treatment outcomes [1].

The role played by metabolic determinants and anthropometric factors in epithelial ovarian cancer (EOC) represents an increasingly attractive area of investigation. Body mass index (BMI) is by far the most commonly assessed anthropometric indicator in association with estimates of ovarian cancer risk and/or mortality. Epidemiologic evidence from large prospective cohort studies generally [2-3], although not exclusively [4], supports the role of BMI as a relevant risk factor for EOC. When considered in light of cancer histology and grade, BMI has been positively associated with increased risk in the less common subtypes, i.e., borderline serous, invasive endometrioid and invasive mucinous epithelial ovarian cancers, while no association has emerged with high grade serous invasive cancer [5]. Notwithstanding the well established link between BMI and glucose levels, there is limited evidence concerning the simultaneous consideration of anthropometric indicators and biomarkers of systemic energetic metabolism in studies aimed at risk assessment for EOC. To our knowledge, the only available data are from a large cohort including 287,320 women. The authors found no evidence in support of the overall association between risk of developing EOC and metabolic syndrome defined based on the standardized sum of pre-set scores for BMI, blood pressure, glucose, cholesterol and triglycerides. Conversely, women with metabolic syndrome showed a higher risk of death by the disease if younger than 50 years, while increasing levels of BMI conferred increased risk of death by ovarian cancer in women aged 50 years and older. No evidence emerged concerning the association between fasting glucose and ovarian cancer risk [6].

Growing data from the preclinical setting provide a plausible rationale on how metabolic and anthropometric determinants may be placed within pathways mechanistically involved in EOC development and progression. An emerging view of carcinogenesis relies on the importance of tumour environment in cancer development. Deregulation in glucose metabolism may have significant effects on the onset and evolution of EOC. The underlying mechanisms have been widely explored at the cellular level, whereas there is still paucity of data from in vivo experiments and from the clinical setting. Indirect evidence on glucose metabolism and EOC also stems from the inveterate use of a number of antihyperglycemic agents currently available for blood glucose control in patients with metabolic disturbances. Due to their ability to impair glucose availability to the tumour, antihyperglycemic drugs such as metformin have been increasingly explored for their potentials as anticancer agents. These drugs have also been shown to have direct effects on metabolic and signaling pathways not necessarily mediated by their action on glucose levels and availability. Evidence from clinical studies is particularly suggestive in diabetic women with ovarian cancer. In these patients, the use of metformin is associated with a reduced risk of cancer and improved survival [7-11].

On this basis, we sought to explore the association between pre-treatment fasting glucose, considered as a proxy for systemic energy metabolism, and widely recognized prognostic factors such as FIGO stage and grade at diagnosis in a historic cohort of patients with EOC for whom data were available for the variables of interest. The impact of anthropometric determinants on the association under evaluation was also assessed in a subgroup of patients for whom BMI data had been collected at baseline.

\section{Methods}

The present analysis includes data on 147 patients with clinically annotated EOC diagnosed and/or treated at the Regina Elena National Cancer Institute of Rome (Central Italy) between May 2005 
and September 2013. To the purpose of the present study, a trained research assistant retrieved medical records on patient-and cancer-related features. Data on fasting glucose were considered suitable for our analysis if all the following conditions were satisfied: 1. Glucose levels had been assessed at baseline, that is, prior to any therapeutic procedures; 2 . Blood drawing had occurred in overnight fasting conditions and in the time window between 7.00 and 10.00 AM; 3. The assessment had been performed at the institutional laboratories where glucose levels had been assessed by hexokinase reagent using a Cobas analyzer and a (Roche Diagnostics). Abnormalities in glucose metabolisms were identified based on the reported use of antidiabetic medications and/or fasting plasma glucose levels greater than $126 \mathrm{mg} / \mathrm{dl}$ [12]. Cancer stage was codified according to the FIGO staging system for ovarian cancer based on the results of the diagnostic workup, while tumour histology and grade were locally assessed by an expert pathologist. Data on demographics were obtained through cross linking to the institutional platform for administrative records, i.e., hospital discharge records. When available, BMI data were integrally extracted from the clinical records or computed as weight in kilograms divided by the square of height in meters.

\section{Statistical analysis}

Distributions were examined and descriptive statistics computed for all the variables of interest. The characteristics of the study participants were reported for the entire historic cohort ( $\mathrm{N}=147)$. Fasting glucose was addressed as a categorical variable whose modalities were defined upon the median value computed in the study population, i.e., $89 \mathrm{mg} / \mathrm{dl}$. Means and standard deviations were used for continuous data while frequencies and percentage values for categorical data. Existing differences between mean values were evaluated using the T-Student or One Way Anova test according to the number (2 or more) of groups compared. We used the Pearson's Chi-squared test of independence or Fisher's exact test (2-tailed) to assess the relationship between categorical variables. We tested several variables for association with FIGO stage and tumour grade using univariate logistic regression analysis. Multivariate models were then built by including those factors testing significant at the univariate analysis and/or for which evidence of a putative role on the association of interest was supported by the available literature (e.g. menopausal status, histology). Menopausal status was self reported. To the purposes of our analysis, the histologic subtype was addressed as a categorical variable including two modalities only, i.e., serous vs non-serous EOC. This was due to the par- ticularly limited number of non-serous EOC which all converged into a unique category finally including 17 cases. Given the prognostic role of diabetes mellitus in EOC, in a subsequent round of analyses, patients with abnormalities in glucose metabolism were identified and excluded [10,12]. Data on BMI were exclusively available for 70 EOC patients. In this subset, we validated the results from the overall cohort according to same statistical and methodological approach. We considered $\mathrm{p}$ values less than 0.05 statistically significant. All statistical analyses were performed with the SPSS statistical software version 21 (SPSS inc., Chicago, IL).

\section{Results}

The descriptive characteristics of the overall cohort are reported in table 1 . Means and standard deviations for age at diagnosis (years) and fasting glucose $(\mathrm{mg} / \mathrm{dl})$ were $57.5( \pm 11.6)$ and $93.6( \pm 20.5)$, respectively. As expected, the vast majority of our patients were diagnosed with a serous histological subtype and fell in the highest grade category.

Table 1. Descriptive characteristics of the study participants (N:147).

\begin{tabular}{lll}
\hline & & mean \pm SD \\
\hline Age (years) & & $57.5 \pm 11.6$ \\
1Fasting Glucose & & $93.6 \pm 20.5$ \\
\multicolumn{1}{l}{ Histologic Subtype } & $\mathrm{N}(\%)$ \\
& Serous & $130(88.4)$ \\
& Poorly differentiated & $4(2.7)$ \\
& Endometriod & $1(0.7)$ \\
& Clear Cell & $1(0.7)$ \\
& Other & $11(7.5)$ \\
${ }^{2}$ FIGO stage & & \\
& I & $22(15.0)$ \\
& II & $16(11.0)$ \\
Tumour Grade & III-IV & $109(74.0)$ \\
& & \\
& I-II & $42(28.6)$ \\
& III & $105(71.4)$ \\
\hline
\end{tabular}

${ }^{1} \mathrm{mg} / \mathrm{dl}$ : milligrams/deciliter; ${ }^{2}$ FIGO: International Federation of Gynecology and Obstetrics

In table 2, FIGO stage is reported by categories of pre-treatment fasting glucose defined upon the median value for this study population, i.e., $89.0 \mathrm{mg} / \mathrm{dl}$. Women whose levels of glycemia were in the lowest category were significantly more likely to be affected by a stage III-IV tumour compared with their counterpart in the highest glycemic category (81.3 vs $66.7 \%, p=0.021)$. Conversely, tumour grade seemed not to be affected by baseline fasting glucose $(p=0.876)$ (table 3 ). The association between fasting glucose and FIGO stage was confirmed in a subset of 70 patients for whom BMI data were available ( 81.5 vs $55.8 \%$, 
$\mathrm{p}=0.049$, table 4 ) and remained significant when tested in regression models including BMI (OR: 0.28 95\% CI 0.086-0.89, p: 0.031). Univariate analysis showed confirmatory findings for the association of fasting glucose and FIGO stage (OR: 0.46, 95\% CI: 0.21-0.98, $\mathrm{p}=0.045$ ), while no significant evidence emerged concerning a putative role of age at diagnosis, histological subtype and menopause (table 5). To assess whether the associations tested were at least partly driven by the presence of patients with abnormalities in glucose metabolism, the entire set of analysis was repeated following exclusion of ten patients from the original cohort. The results obtained did not differ from those previously observed at any extent (supplementary table 1).

Table 2. Tumour FIGO stage by categories of fasting glucose (N:147).

\begin{tabular}{cllll}
\hline & \multicolumn{4}{c}{ 2FIGO stage } \\
\cline { 2 - 5 } & $\mathrm{I}$ & $\mathrm{II}$ & $\mathrm{III}-\mathrm{IV}$ & Chi2 \\
\cline { 2 - 5 } & $\mathrm{N}=22$ & $\mathrm{~N}=16$ & $\mathrm{~N}=109$ & $\mathrm{p}$-value \\
\cline { 2 - 5 } & $\mathrm{n}(\%)$ & $\mathrm{n}(\%)$ & $\mathrm{n}(\%)$ & \\
\hline 1Fasting Glucose & & & & \\
$\leq 189$ & $11(14.7)$ & $3(4.0)$ & $61(81.3)$ & 0.021 \\
$>89$ & $11(15.3)$ & $13(18.1)$ & $48(66.7)$ & \\
\hline
\end{tabular}

${ }^{1}$ in mg/dl: milligrams/deciliter; ${ }^{2}$ International Federation of Gynecology and Obstetrics

Table 3. Tumour grade by categories of fasting glucose $(N=147)$.

\begin{tabular}{rlll}
\hline & \multicolumn{3}{c}{${ }^{2} \mathrm{G}$} \\
\cline { 2 - 4 } & $\mathrm{G} 1-\mathrm{G} 2$ & $\mathrm{G} 3$ & Chi2 \\
\cline { 2 - 4 } & $\mathrm{N}=42$ & $\mathrm{~N}=105$ & p-value \\
\cline { 2 - 4 } & $\mathrm{n}(\%)$ & $\mathrm{n}(\%)$ & \\
\hline Fasting Glucose & $21(28.0)$ & $54(72.0)$ & 0.876 \\
$>89$ & $21(29.2)$ & $51(70.8)$ & \\
\hline
\end{tabular}

${ }^{1} \mathrm{mg} /$ dl: milligrams/deciliter; ${ }^{2}$ Grade

Table 4. Tumour FIGO stage by categories of fasting glucose in epithelial ovarian cancer patients with available data on body mass index ( $N: 70)$.

\begin{tabular}{cllll}
\hline & \multicolumn{4}{c}{ 1FIGO } \\
\cline { 2 - 5 } & $\mathrm{I}$ & $\mathrm{II}$ & $\mathrm{III}-\mathrm{IV}$ & Chi2 \\
\cline { 2 - 5 } & $\mathrm{N}=13$ & $\mathrm{~N}=11$ & $\mathrm{~N}=46$ & $\mathrm{p}$-value \\
\cline { 2 - 5 } & $\mathrm{n}(\%)$ & $\mathrm{n}(\%)$ & $\mathrm{n}(\%)$ & \\
\hline Glycaemia at the baseline & & & & \\
$\leq 189$ & $4(14.8)$ & $1(3.7)$ & $22(81.5)$ & 0.049 \\
$>89$ & $9(20.9)$ & $10(23.3)$ & $24(55.8)$ & \\
\hline
\end{tabular}

${ }^{1}$ International Federation of Gynecology and Obstetrics; ${ }^{2} \mathrm{mg} / \mathrm{dl}$ : milligrams/deciliter

\section{Discussion}

We sought to verify the association of proxies of systemic energy metabolism and renown disease-related prognostic factors in a historic cohort of 147 women diagnosed with EOC at the Regina Elena
National Cancer Institute between May 2005 and September 2013. The exposure of interest was pre-treatment fasting glucose, while tumour stage and grade were identified among the prognostic determinants for which medical records were available. We found novel evidence of a significant inverse association between fasting glycemia and FIGO stage at diagnosis in the overall study cohort. Results were confirmed in subgroup analysis including patients with anthropometric data and remained statistically significant in multivariate models including BMI. Conversely, no evidence of an influence of glucose levels on tumour grade emerged either in the entire cohort or in subgroup analysis. No significant evidence was found in support of any associations between fasting glucose and age at diagnosis, histological subtype and menopause. Our results were not affected by the exclusion of 10 patients identified on the basis of glucose dismetabolisms as defined by altered glucose levels and/or reported use of antidiabetic drugs.

Table 5. Univariate analysis of factors associated with FIGO stage at diagnosis $(\mathrm{N}=147)$.

\begin{tabular}{|c|c|c|c|c|}
\hline & & ${ }^{2} \mathrm{OR}$ & $395 \% \mathrm{CI}$ & p-value \\
\hline \multirow[t]{2}{*}{ 1Fasting Glucose } & $\leq 89$ & 1 & & \\
\hline & $>89$ & 0.46 & $0.25-0.98$ & 0.045 \\
\hline Age at Diagnosis & & 0.98 & $0.95-1.01$ & 0.281 \\
\hline \multirow{2}{*}{ Histological Subtype } & Other & 1 & & \\
\hline & Serous & 0.58 & $0.16-2.15$ & 0.416 \\
\hline \multirow[t]{2}{*}{ Menopause } & Premenopausal & 1 & & \\
\hline & Postmenopausal & 0.70 & $0.29-1.70$ & 0.434 \\
\hline
\end{tabular}

Our first time finding of an inverse association between pre-treatment glucose levels and FIGO stage in EOC is of non univocal interpretation. In non diabetic patients with advanced stage tumours, glucose levels in the lower range may be a sign of systemic impairment with reflections on liver functions and altered gluconeogenesis. This is generally accompanied by loss of weight, with a basically low BMI and scarce performance status. On this basis, in a set of adjunctive analyses, we verified whether FIGO stage was affected by BMI and performance status (PS) as evaluated based on the Eastern Cooperative Oncology Group (ECOG) scale. Data were available only for a subgroup of the original cohort $(n=70)$. No evidence emerged concerning any association between ECOG performance status and stage at diagnosis. In more detail, as a result of the clinical evaluation performed at baseline, these patients had been all assigned to the first two categories of the ECOG scale (data available upon request). Similarly, our ad hoc analysis did not 
support any association between BMI and FIGO stage (supplementary table 2). In addition, since our results were not affected by the exclusion of 10 women with glucose dismetabolism (supplementary table 1), we may exclude that our results were driven by the inappropriate glycemic control occurring in this subset of patients.

As an alternative hypothesis, glucose levels in the lower range might represent indirect evidence of accelerated glucose transport and increase glucose uptake related to enhanced expression of the protein transporter protein 1 (GLUT1) in EOC with advanced FIGO stage at diagnosis. GLUT 1 is a hexose transporter ascribed to the GLUT family, which includes fourteen facilitative transporters working along the glucose concentration gradient [13-14]. Among them, GLUT1 is the most widely expressed and is involved in maintaining basal glucose transport in several normal cell types and cancer cells [15-20]. The existence of a significant correlation between increased expression of GLUT1 in serous adenocarcinomas of the ovary and FIGO stage has been consistently shown in previous research. Cai and colleagues worked on two hundred formalin-fixed, paraffin-embedded sections from 40 normal ovarian tissues, 40 serous cystoadenomas, 40 borderline serous cystoadenomas and 80 serous cystoadenocarcinomas. In their study, results from immunostaining with rabbit anti-GLUT1 polyclonal antibody showed increased expression of GLUT1 and p63, a homolog of p53, in cystoadenocarcinomas compared to benign serous and borderline cystoadenomas. In addition, overexpression of GLUT1 and p63 were associated with the FIGO stage and metastasis of the tumors [21]. Similar results were reported by Shao and colleagues [22]. Hitherto, no study has evaluated whether in high FIGO stage EOC the increased expression of GLUT1 had any sort of resonance on the expression of this same receptor in other districts and/or at the systemic level. GLUT1 is physiologically over-expressed at the interface with cells or tissues with a glucose-driven metabolism, e.g. red blood cells membrane, perineum of the peripheral nerves. On a still highly speculative reasoning, we might hypothesize a sort of homeostatic equilibrium and the occurrence of consensual changes in the expression of this receptor at a multidistrict level following metabolic adaption of the ovary to the enhanced energy necessities dictated by the carcinogenetic process.

When moving to the opposite side of a normal glycemic interval, the evidence of glucose levels in the higher range of fasting glucose in patients diagnosed with low FIGO stage EOC might encourage investigating the gynecological district for screening purposes in non-diabetic, asymptomatic women. Under the putative umbrella of confirmatory results from further studies, this approach might further inform the allocation of these women to categories differing by risk of EOC development. Given its favorable cost-benefit balance, the evaluation of indicators of the systemic metabolic asset jointly with anthropometric measurements might be proposed as an easy and efficacious integration to the most updated, though still not univocally clarified, orientations and guidelines for ovarian cancer screening [23-24].

Our results are barely comparable to previous findings. To the best of our knowledge, no prior studies have addressed the association between fasting glucose and EOC cancer stage at diagnosis, while glycemia has been explored in relation to risk of EOC development in one single study with negative findings [7]. The literature concerning the impact of the exposure of interest on treatment outcomes in EOC patients is somewhat less scant. Lamkin and colleagues tested the hypothesis of higher pre-surgical glucose levels associated with shorter time to recurrence and disease-specific survival in 74 patients with ovarian cancer and used an independent set of 125 ovarian cancer cases for results validation. The authors observed a relationship between higher pre-surgical glucose levels in ovarian cancer patients and decreased survival time and disease free interval. Confirmatory results came from the validation set. However, a not negligible limitation to the generalizability of this study results was introduced by glucose assessment in plasma samples collected in non fasting conditions. As clearly stated by the authors themselves, the measurement of glucose levels without standardizing for fasting duration introduced a considerable source of variability, particularly when working on a mixed set including both diabetic and non-diabetic patients. In addition, postload plasma glucose and fasting glucose were proven to differentially impact cancer mortality [25-27].

This invites further caution in comparing results from our study with the findings from the work of Lamkin and colleagues.

Highly standardized operative conditions at blood collection and processing, along with the exclusive use of the institutional certified laboratories of clinical pathology for fasting glucose measurement, represent the major strengths of this study. Similarly, standardized procedures were applied at the time of tissue handling and specimen preparation, which both occurred under the supervision of a pathologist with renown expertise in gynecological cancers. The relatively large historic cohort and the simplicity of the scientific hypothesis stated and methods applied might add some value to our work.

Among the main limitations, it is noteworthy the 
lack of BMI data for the entire cohort. Missing values for a given exposure are extremely common when relying on data primarily collected for clinical purposes. In addition, among the indicators of the systemic metabolic asset closely related to glucose metabolism, we exclusively focused on fasting glucose while not including in our analysis potentially relevant markers, such as baseline insulin, insulin-like growth factor-I (IGF-I) and insulin-like growth factors. Unfortunately, serum measurements of such determinants were not available for these patients since, differently from the assessment of glycemia, their assessment is not encompassed into clinical routine.

\section{Conclusions}

In summary, we provide novel evidence of the association between pre-treatment fasting glucose and FIGO stage at diagnosis in a historic cohort of 147 women diagnosed with EOC at the Regina Elena Cancer Institute of Rome. Although such evidence is non univocally interpretable, it might be in key with the hypothesis of a role of metabolic influxes on renown prognostic factors, such as FIGO stage, in EOC. This latter hypothesis fuels active research in a still largely unexplored and extremely promising area of investigation in ovarian and other cancers [28-34]. Within this pipeline, future work from our group will be bidirectionally oriented. We aim to confirm our results in larger and prospectively conducted studies with both a districtual and systemic focus, i.e., focused on the evaluation of the role of determinants of glucose metabolism not only at an ovarian tissue level, but also on blood samples collected for pre-defined study purposes. Our approach will be effectively enriched by the use of immunophenotipying and oncogenomic techniques which will be coupled to the classical morphologic characterization. This strategy will hopefully contribute mechanistic insights pertinent to the molecular players and related pathways underlying the potential role of metabolic determinants on risk stratification and treatment outcomes in EOC patients.

\section{Supplementary Material}

Supplementary Tables 1-2.

http://www.jcancer.org/v07p0516s1.pdf

\section{Abbreviations}

BMI: Body mass index scale

ECOG: Eastern Cooperative Oncology Group

EOC: Epithelial ovarian cancer

FIGO: International Federation Gynecology and Obstetrics
IGF-I: Insulin-like growth factor-I

$\mathrm{mg} / \mathrm{dl}$ : Milligram per deciliter

SPSS: Statistical Package for Social Science

\section{Acknowledgments}

We thank Dr Anamaria Edliska for data management and technical assistance and Dr Tania Merlino for English revisions.

\section{Authors' contribution}

PV and LP: data acquisition, critical interpretation of the study results, manuscript drafting; LC, LDL, AG, and EV: data acquisition, critical revision for important intellectual contents; CM, AA, GD, DS, MV, AG, EV, GC, CV, RK, AM, MC and BA: data acquisition; PM, FT and AG: critical revision for important intellectual contents; FS data acquisition and analysis; MMS and MB: study conception and design, analysis planning, results interpretation and manuscript writing.

All authors read and approved the final manuscript.

\section{Competing Interests}

The authors have declared that no competing interest exists.

\section{References}

1. Ferlay J, Steliarova-Foucher E, Lortet-Tieulent J, et al. Cancer incidence and mortality patterns in Europe: estimates for 40 countries in 2012. Eur J Cancer. 2013;49:1374-1403.

2. Schouten L, Rivera C, Hunter D, et al. Height, body mass index, and ovarian cancer: a pooled analysis of 12 cohort studies. Cancer Epidemiol, Biomarkers Prev. 2008;17:902-12.

3. Lahmann P, Cust A, Friedenreich C, et al. Anthropometric measures and epithelial ovarian cancer risk in the European Prospective Investigation into Cancer and Nutrition. IJC. 2010;126:2404-15.

4. Kotsopoulos J, Baer H, Tworoger S. Anthropometric Measures and Risk of Epithelial Ovarian Cancer: Results From the Nurses' Health Study. Obesity (Silver Spring). 2010;18:1625-31.

5. Olsen C, Nagle C, Whiteman D, et al. Obesity and risk of ovarian cancer subtypes: evidence from the Ovarian Cancer Association Consortium. Endocr Relat Cancer. 2013;20:251-62.

6. Bjørge T, Lukanova A, Tretli S, et al. Metabolic risk factors and ovarian cancer in the Metabolic Syndrome and Cancer project. I Journal Epy. 2011;40: 1667-77.

7. Kellenberger L, Bruin J, Greenaway J, et al. The Role of Dysregulated GlucoseMetabolism in Epithelial Ovarian Cancer. J Oncol. 2010: 514310.

8. Febbraro T, Lengyel E, Romero IL. Old drug, new trick: repurposing metformin for gynecologic cancers? Gynecol Oncol. 2014;135:614-21.

9. Bodmer M, Becker C, Meier C, et al. Use of metformin and the risk of ovarian cancer: A case-control analysis. Gynecol Oncol. 2011;123: 200-204.

10. Romero I, McCormick A, McEwen K, et al. Relationship of type II diabetes and metformin use to ovarian cancer progression, survival and chemosensitivity. Obstet Gynecol. 2012;119: 61-67.

11. Kumar S, Meuter A, Thapa P, et al. Metformin intake is associated with better survival in ovarian cancer: A case-control study. Cancer. 2013:119:555-562.

12. American Diabetes Association. American Diabetes Association Clinical Practice Recommendations. Diabetes Care. 2008;31:S1-S108.

13. Zhao FQ, Keating AF. Functional properties and genomics of glucose transporters. Curr Genomics. 2007;8:113-128.

14. Manolescu A, Witkowska K, Kinnaird A, et al. Facilitated hexose transporters: new perspectives on form and function. Physiology. 2007;22:234-240.

15. Younes M, Brown R, Stephenson M, et al. Overexpression of Glut1 and Glut3 in stage I non small cell lung carcinoma is associated with poor survival. Cancer. 1997;80:1046-1051.

16. Kang S, Chun Y, Hur M, et al. Clinical significance of glucose transporter 1 (GLUT1) expression in human breast carcinoma. Jpn J Cancer Res. 2002;93:1123-1128 
17. Boado RJ, Black KL, Pardridge WM. Gene expression of GLUT3 and GLUT1 glucose transporters in human brain tumors. Brain Res Mol Brain Res 1994;27:51-57.

18. Amann T, Maegdefrau U, Hartmann A, et al. GLUT1 expression is increased in hepatocellular carcinoma and promotes tumorigenesis. Am J Pathol. 2009;174:1544-1552.

19. Godoy A, Ulloa V, Rodriguez F, et al. Differential subcellular distribution of glucose transporters GLUT1-6 and GLUT9 in human cancer: ultrastructural localization of GLUT1 and GLUT5 in breast tumor tissues. J Cell Physiol. 2006;207:614-627.

20. Younes M, Brown R, Mody D, et al. GLUT1 expression in human breast carcinoma: correlation with known prognostic markers. Anticancer Res. 1995;15:2895-2898.

21. Cai Y, Zhai J, Feng B, et al. Expression of glucose transporter protein 1 and p63 in serous ovarian tumor. J Obstet Gynaecol Res. 2014;40:1925-30.

22. Shao S, Cai Y, Wang Q, et al. Expression of GLUT-1, p63 and DNA-Pkcs in serous ovarian tumors and their significance. Zhonghua Zhong Liu Za Zhi. 2007;29:697-700.

23. Wardle J, Robb K, Vernon S, et al. Screening for prevention and early diagnosis of cancer. J Am Psychol. 2015;70:119-33.

24. Gentry-Maharaj A, Menon U. Screening for ovarian cancer in the general population. Best Practice \& Research Clinical Obstetrics \& Gynaecology. 2012;26:243-256.

25. Lamkin D, Spitz D, Shahzad M, et al. Glucose as a prognostic factor in ovarian carcinoma. Cancer. 2009;115: 1021-7.

26. Gapstur S, Gann P, Lowe W, et al. Abnormal glucose metabolism and pancreatic cancer mortality. JAMA. 2000;283:2552-2558.

27. Shaw J, Hodge A, de Courten M, et al. Isolated post-challenge hyperglycemia confirmed as a risk factor for mortality. Diabetologia. 1999;42:1050-1054.

28. Guo T, Chen T, Gu C, et al. Genetic and molecular analyses reveal G6PC as a key element connecting glucose metabolism and cell cycle control in ovarian cancer. Tumour Biol. 2015; Epub ahead of print.

29. Koti M, Gooding R, Nuin P, et al. Identification of the IGF1/PI3K/NF KB/ERK gene signalling networks associated with chemotherapy resistance and treatment response in high-grade serous epithelial ovarian cancer. BMC Cancer. 2013;13:549.

30. Krzeslak A, Wojcik-Krowiranda K, Forma E, et al. Expression of GLUT1 and GLUT3 glucose transporters in endometrial and breast cancers. Pathol Oncol Res. 2012;18:721-8.

31. Young C, Lewis A, Rudolph M, et al. Modulation of glucose transporter 1 (GLUT1) expression levels alters mouse mammary tumor cell growth in vitro and in vivo. PLoS One. 2011;6:e23205.

32. Barba M, Sperati F, Stranges S, et al. Fasting glucose and treatment outcome in breast and colorectal cancer patients treated with targeted agents: results from a historic cohort. Ann Oncol. 2012;23:1838-45.

33. Vici P, Sperati F, Maugeri-Saccà M, et al. p53 status as effect modifier of the association between pre-treatment fasting glucose and breast cancer outcomes in non diabetic, HER2 positive patients treated with trastuzumab. Oncotarget. 2014;5:10382-92.

34. Nagle CM, Dixon SC, Jensen A. Obesity and survival among women with ovarian cancer: results from the Ovarian Cancer Association Consortium. Br J Cancer. 2015;Epub ahead of print. 